to previous estimates of helium abundance in $\mathrm{H}$ II galaxies.

Ferland has derived new values of $Y_{\mathrm{p}}$ based on measurements of the $5,876 \AA$ intensities for several galaxies, incorporating quoted electron densities, and in three cases these are as low as $0.19-0.20$. If correct, these values would raise grave doubts about the standard model. However, for easily indentifiable reasons they are all either incorrect or doubtful.

Although he has exaggerated the drama of the situation, Ferland has performed a valuable service in drawing attention to the importance of the collisional contribution to helium lines in $\mathrm{H}_{\text {II }}$ galaxies and has demonstrated that some downward correction to the deduced helium abundance is needed. Applying such a correction to our published data, we find $Y_{\mathrm{p}}=$ $0.232 \pm 0.004(1 \sigma)$, very close to previous estimates by Peimbert".

This value is $2 \sigma$ below the bare minimum requirements of SBBN with the limit on $\mathrm{D}+{ }^{3} \mathrm{He}$, leaving three alternatives: (1) there are further systematic errors (not yet identified) in the determination of $Y_{\mathrm{p}}$ from $\mathrm{H}$ II galaxies; (2) the $\mathrm{D}+{ }^{3} \mathrm{He}$ argument is wrong because of unsafe assumptions about galactic chemical evolution, for example there could have been a

NEWSANDVIEWS

greater number of massive stars in the past than allowed for ${ }^{10}$ which would predict a large deuterium abundance in the Lyman$\alpha$ forest clouds seen in absorption in the spectra of quasars, as these clouds are believed to represent material that has undergone relatively little processing since the Big Bang; (3) SBBN needs modification, possibly because $N_{v}=2$ and not 3 (that is, the $\tau$ neutrino is massive) possibly because of fluctuations, or possibly for some other reason. Further developments are eagerly awaited.

Yang, J., Turner, M. S., Steigman, G., Schramm, D. N. \& Olive, K. A. Astrophys. J. 281, 493-511 (1984).

2. Boesgaard, A. M. \& Steigman, G. A. Rev. Astr. Astrophys. 23, 319-378 (1985)

3. Sale, K. E. \& Matthews, G. J. Astrophys. J. 309, L1-L4 (1986)

4. Peimbert, M. \& Torres-Peimbert, S. Astrophys. J. 193, 327-333 (1974)

5. Pagel, B. E. J., Terlevich, R. \& Melnick, J. Publ. Astr. Soc. Pacific. 98, 1005-1011 (1986)

6. Brocklehurst, M. Mon. Not. R. astr. Soc. 157, 211-227 (1972).

Berrington, K. A., Burke, P. G., Freitas, L. \& Kingston, A. E. J. Phys. B18, 4135-4147 (1985).

Ferland, G. J. Astrophys. J. 310, L67-L70 (1986)

9. Peimbert, M. in Star Forming Dwarf Galaxies (eds Kunth, D., Thuan, T.X. \& Tran Thanh Van, J.) 403-415 (Frontières, Paris, 1985).

10. Larson, R. B. Mon. Not. R. astr. Soc. 218, 409-428(1986)

B. E. J. Pagel is at the Royal Greenwich Observatory, Herstmonceux Castle, Hailsham, East Sussex BN27 1RP, UK.

\title{
Protein engineering
}

\section{Inhibition into activation}

\section{Max Perutz}

ON page 811 of this issue', Lau and Fersht report that directed mutagenesis of the enzyme phosphofructokinase (PFK) alters its properties in a manner not observed before in an allosteric protein, and apparently in conflict with allosteric theory. PFK, a tetrameric enzyme, catalyses the phosphorylation of fructose-6phosphate (F6P) by ATP to form fructose1,6-phosphate and ADP. The reaction is cooperative with respect to $\mathrm{F} 6 \mathrm{P}$ and is inhibited by phosphoenolpyruvate (PEP).

$\mathrm{X}$-ray analysis has shown that cooperativity is caused by an equilibrium between two alternative quaternary protein structures. Two catalytic sites of PFK are located at one pair of symmetry-related subunit interfaces; two regulatory sites occupy another such pair of interfaces ${ }^{2}$. Precise knowledge of the stereochemistry at each of these sites has opened the way to the exploration of the catalytic mechanism by directed mutagenesis, in which single amino-acid residues can be replaced. Amino-acid replacements in the catalytic sites have provided proof that an aspartate close to the $1-\mathrm{OH}$ of the bound F6P activates its nucleophilic attack on the $\gamma$-phosphate of ATP $^{3}$. Directed mutagenesis in the regulatory sites, reported by Lau and Fersht in this issue ${ }^{\prime}$, shows that the replacement Glu $187 \rightarrow$ Ala changes PEP from an inhibitor to an activator of the reaction of F6P with ATP, but at the same time reduces the maximal catalytic rate of which the enzyme is capable. How can this be reconciled with allosteric theory ${ }^{4}$ ?

According to this theory, proteins exhibiting cooperative behaviour are oligomers consisting of several subunits. These oligomers may exist in two or at least two different states that differ in their affinities for substrate or in their catalytic rates. Structurally, the states should differ in the arrangement of the subunits and the distribution and/or energy of the bonds between them, and therefore also in the constraints imposed on them. The stronger bonds constrain the protein to a tense $(T)$ state with low substrate affinity or catalytic activity; the weaker bonds allow the protein to relax to the $R$ state in which the subunits develop their full substrate affinity or catalytic activity. Allosteric effectors act by altering the equilibrium between the alternative states. In its mathematical form the theory can account for the properties of such proteins in terms of only three variables: the ligand equilibrium constants $K_{T}$ and $K_{R}$ (or $V_{\max }(T)$ and $\left.V_{\max }(R)\right)$, and $L=[T] /[R]$ in the absence of substrate. The values of $K$ and $V_{\max }$ are independent of the number of substrate molecules bound to the $T$ or $R$ states. Allosteric effectors would alter $L$, and be without influence on $K_{T}$ and $K_{R}$. This formulation has led to Monod, Wyman and Changeux's theory being referred to (and frequently attacked) as the 'two-state model', but the text of their paper ${ }^{4}$ shows that they sensibly restricted their mathematical formulation to the simplest case, while being aware that the behaviour of real systems might be more complex.

Although allosteric theory was proposed more than 20 years ago, its predictions have been tested in detail only for the three allosteric proteins whose structures have been determined: haemoglobin, aspartate transcarbamylase and PFK. X-ray analysis confirms the existence of each of these oligomeric proteins in two alternative quaternary structures that differ in the arrangement of their subunits. The prediction of additional bonds between the subunits in the $T$ structure has been confirmed in haemoglobin, but may not apply to the two enzymes, whose active sites actually lie at the subunit interfaces and must be maintained by bonds between them. Here only the distribution of the bonds may differ. In haemoglobin and PFK allosteric effectors are known to add to the constraints of the $T$ structure by forming bonds between the subunits. These findings are in accord with the simple allosteric theory, but the thermodynamic behaviour of haemoglobin has turned out to be more complex. $K_{T}$ varies over the equivalent of $2 \mathrm{kcal} \mathrm{mol}^{-1}$ haem, depending on $p \mathrm{H},\left[\mathrm{Cl}^{-}\right]$and allosteric effector concentration ${ }^{s}$. Thus, $K_{T}$ depends on the constraints of the $T$ structure, which can therefore assume an infinity of thermodynamic states.

The mutant of PFK now discovered by Lau and Fersht is unique in switching the affinity of an allosteric effector from the $T$ to the $R$ structure. On the other hand, the reduction in the maximal catalytic rate is what one would expect if the binding of an allosteric effector to the $R$ structure introduced bonds between the subunits which distorted the catalytic sites. In PFK, the active site lies between two domains of one subunit and one domain of a neighbouring subunit; catalytic activity may be influenced by rearrangements of the subunits as well as of the domains and also by changes in tertiary structure within the domains. X-ray analysis of the mutant enzyme should reveal which of these is dominant.

1. Lau, F.T.-K. \& Fersht, A.R. Nature 326, 811-812 (1987).

. Evans, P.R., Farrants, G.W. \& Hudson, P.J. Phil. Tran R. Soc. B293, 53-62 (1981)

Hellinga, H.W. \& Evans, P.R. Nature (in the press)

4. Monod, J., Wyman, J. \& Changeux, J.-P. J. molec. Biol. 12, 88-118 (1965).

5. Imai, K. Allosteric Effects in Haemoglobin (Cambridge University Press, 1982).

Max Perutz is at the MRC Laboratory of Molecular Biology, Cambridge CB2 2QH, UK. 